\title{
HHV8-Positive Germinotropic Lymphoproliferative Disorder
}

National Cancer Institute

\section{Source}

National Cancer Institute. HHV8-Positive Germinotropic Lymphoproliferative Disorder. NCl Thesaurus. Code C150405.

A monotypic HHV8-positive lymphoproliferative disorder that usually occurs in HIVnegative individuals. It is characterized by the presence of HHV8-positive plasmablasts partially or completely replacing germinal centers. Coinfection with EBV is characteristic. In most cases, there is a favorable response to chemotherapy or radiation. (WHO 2017) 